\title{
Open-label trial with artemether-lumefantrine against uncomplicated Plasmodium falciparum malaria three years after its broad introduction in Jimma Zone, Ethiopia
}

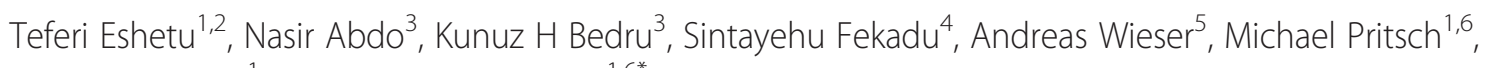
Thomas Löscher ${ }^{1}$ and Nicole Berens-Riha ${ }^{1,6^{*}}$

\begin{abstract}
Background: In Jimma Zone, Ethiopia, the first-line treatment of uncomplicated falciparum malaria has been changed from sulphadoxine-pyrimethamine (SP) to artemether-lumefantrine (AL) in 2006. The objective of this study was to assess the effectiveness of AL in Jimma Zone two to three years after its broad introduction.

Methods: An open-label, single-arm, 42-day study of AL against falciparum malaria was conducted in four areas with moderate transmission in Jimma Zone between November 2008 and January 2009 and between August and December 2009. Patients (one-81 years) with uncomplicated Plasmodium falciparum mono-infection were consecutively enrolled. Follow-up visits were at day 2, 3, 7, 28 and 42 or any other day if symptoms reoccurred. Primary and secondary endpoints were PCR-corrected and uncorrected cure rates (molecular differentiation between recrudescence and re-infection) on days 28 and 42 . Other secondary endpoints were gametocytaemia at day 7 and day 28, parasitaemia at day 2 and 3, and re-infection rates at day 28 and day 42 .

Results: Of 348 enrolled patients, 313 and 301 completed follow-up at day 28 and at day 42, respectively. No early treatment failure occurred. For per protocol analysis, PCR-uncorrected cure rates at day 28 and 42 were $99.1 \%$ (95\% Cl 98.0-100.0) and 91.1\% (95\% Cl 87.9-94.3), respectively. PCR-corrected cure rates at day 28 and 42 were $99.4 \%$ (95\% Cl 98.5-100.0) and 94.7\% (95\% Cl 92.2-97.2), respectively. PCR-corrected cure rate at day 42 for children $\leq 5$ years was $90.6 \%$ (95\% Cl 82.4-98.7) only. Adverse events were in general mild to moderate. Incidence of new infections was 3.4\% during 42 days, no new infections with Plasmodium vivax were observed. Microscopically detected gametocytaemia was reduced by $80 \%$ between day 0 and day 7 .

Conclusion: In general, AL was effective and well tolerated in Jimma Zone, Ethiopia. However, the PCR-corrected recrudescence rate per-protocol at day 42 for children $\leq 5$ years was $9.4 \%$. Therefore, further development should be monitored on a regular basis as recommended by WHO.
\end{abstract}

Keywords: Malaria, Plasmodium falciparum, Artemether-Lumefantrine, ACT, Ethiopia

\footnotetext{
*Correspondence: berens@|rz.uni-muenchen.de

'Division of Infectious Diseases and Tropical Medicine, Medical Center of the University of Munich (LMU), Leopoldstrasse 5, 80802 Munich, Germany

${ }^{6} \mathrm{German}$ Centre for Infection Research (DZIF) at LMU, Munich, Germany

Full list of author information is available at the end of the article
} 


\section{Background}

Due to increasing resistance of Plasmodium falciparum against previous anti-malarials, such as chloroquine and sulphadoxine-pyrimethamine (SP), most African countries, including Ethiopia, have changed their national policy in recent years towards first-line treatment with artemisinin-based combination therapy (ACT) [1,2]. ACT produces faster relief of clinical symptoms and parasite clearance in uncomplicated $P$. falciparum malaria than any other currently available anti-malarial drug $[3,4]$. It combines the potential of rapid reduction of the parasite burden with the elimination of remaining parasites due to longer acting partner drugs [5]. ACT seems to be well tolerated as several studies indicate; no severe side effects have been observed, especially for the combination artemether-lumefantrine (AL) [6,7].

Malaria is endemic in approximately $75 \%$ of the national territory of Ethiopia. About 50 million inhabitants are at risk of infection. In 2009, there was a considerable increase of malaria-associated morbidity and mortality in the south and south-west of Ethiopia, including the town of Jimma and its surroundings, with most cases occurring from September to December. Predominant species was $P$. falciparum, but a trend to mixed and mono-infections with Plasmodium vivax was observed $[8,9]$, pers comm with local health centres]. AL was introduced in Ethiopia as first-line treatment for uncomplicated falciparum malaria in 2004 and became available in Jimma Zone in 2006. Since then, AL was provided free of charge at public health centres by the government only.

Increasing recrudescence rates and reduced in vitro response to artemisinins were first reported from some Asian countries [10], where artesunate monotherapy has commonly been used for some decades. Along with sub-therapeutic doses, incomplete treatment courses, substandard and fake artemisinin-based drugs, this constitutes a major risk factor for the development of drug resistance. In 2008, the alarming news of delayed parasite clearance rates after treatment with artesunate monotherapy in Cambodia arrived. Reports on increasing recrudescence and late clearance rates with ACT followed [11-14]. A new definition for artemisinin resistance was introduced by the WHO [10]. Interestingly, slow clearance rates did not seem to be associated with decreased sensitivity to drugs in vitro, but were associated with parasite genetics in Cambodia [15]. Resistance spread on a genetic basis would enormously compromise the recent success of ACT in reducing the incidence of malaria in various regions $[16,17]$. Since the WHO memorandum in 2011, the use of artemisininbased monotherapies is no longer recommended [18].

The broad introduction of ACT in many African countries offers the chance of monitoring drug- resistance development right from the start. Several studies from neighbouring countries and from Ethiopia showed high cure rates for AL and other ACT [19-26]. However, one recent study raised concerns over prolonged clearance rates in Africa [27].

The underlying study was aimed at investigating the clinical and parasitological effectiveness of AL in Jimma Zone two to three years after its broad introduction.

\section{Methods}

\section{Study area and recruitment}

Jimma is the capital of Jimma Zone in Oromiya regional state and is located $355 \mathrm{~km}$ south-west of the Ethiopian capital, Addis Ababa. Jimma Zone and its surroundings are malaria endemic with a low to moderate transmission. The predominant species in most areas is P. falciparum, followed by $P$. vivax. Most cases occur from September to December after the main rainy season from June to $\mathrm{Au}$ gust. Recruitment took place over eight months between November 2008 to January 2009 and August 2009 to December 2009. Study sites were Agaro Health Centre, Jimma Health Centre, Serbo Health Centre, and Asendabo Health Centre (average elevation of study areas between $1,667 \mathrm{~m}$ to $1,772 \mathrm{~m}$ above sea level).

\section{Study design and patients}

The study was an open-label, single-arm, non-supervised trial with standard treatment artemether-lumefantrine in a six-dose, weight-adapted regimen. The study was implemented in routine diagnostic of the health centres. Blood slides were performed from all patients with fever. Only falciparum mono-infection was considered for the study. After microscopic confirmation, patients were informed about the study and screened for eligibility if consent was given.

Inclusion criteria were age above one year, body weight above $5 \mathrm{~kg}$, uncomplicated $P$. falciparum malaria (mono-infection with $P$. falciparum), parasite density $1,000-100,000 / \mu \mathrm{L}$, axillary temperature $\geq 37.5^{\circ} \mathrm{C}$ or recorded history of fever within preceding $24 \mathrm{hrs}$, ability to tolerate oral therapy, informed consent by the patient or the legal representative as well as residency in the study area. Treatment of uncomplicated malaria is on an outpatient basis, therefore, eligibility for the study was limited to residency in walking distance to the health posts or the ability to use public transportation $(<2$ hours of travel time) due to the follow-up schedule of the study.

Exclusion criteria were any anti-malarial treatment within the previous seven days (anti-malarials like chloroquine, AL, SP, quinine and others as well as antibiotics with anti-malarial effects like doxycycline, clindamycine), mixed plasmodial infection, danger signs (inability to drink, repeated vomiting, recent history of 
convulsions, lethargy or unconsciousness) and signs of severe malaria as defined by the WHO [28] or any other known severe underlying disease (eg, cardiac, renal, hepatic diseases, severe malnutrition, known HIV infection). Patients with history of allergy or intolerance against study medications, during pregnancy or lactation were also excluded.

\section{Ethical considerations}

Each patient (or parents/guardians) received oral and written information about the study and signed an informed consent form. Ethical approval was obtained from the Ethical Board of Jimma University, the Ethiopian Federal Ministry of Science and Technology, and from the Ethical Committee of the Faculty of Medicine of the Ludwig-Maximilian-University (LMU) in Germany.

\section{Treatment}

The standard oral regimen of Coartem ${ }^{\circledR}$ (Novartis Pharmaceuticals Corporation, Suffern, New York, USA for Novartis AG, $20 \mathrm{mg}$ artemether/120 mg lumefantrine) was used for treatment which is supplied free of charge by the government at health centres and hospitals. The government is the only legal distributor of AL in Ethiopia. In a drug quality control centre in Addis Ababa, drug tests are performed on a regular basis to exclude fake drugs.

AL was administered as follows: $20 \mathrm{mg}$ of artemether and $120 \mathrm{mg}$ of lumefantrine (children $5-14 \mathrm{~kg}$ ), $40 \mathrm{mg} /$ $240 \mathrm{mg}$ (children 15-24 kg), $60 \mathrm{mg} / 360 \mathrm{mg}$ (children $25-34 \mathrm{~kg}$ ), $80 \mathrm{mg} / 480 \mathrm{mg}$ (adults and children $\geq 35 \mathrm{~kg}$ bwt.), at hrs $0,8,24,36,48$ and 60 (six doses). Patients were instructed to take the doses with fatty food. Full treatment was supplied on admission. The first dose was administered at the health centres by medical staff under supervision. Full re-dosing was performed, if vomiting occurred within 30 mins after the first dose given. If a patient was unable to tolerate the medication, treatment was discontinued and the patient referred to the hospital for intravenous (IV) treatment with quinine. Therapy for early treatment failures would have followed the respective local guidelines for second-line therapy and consisted of IV quinine. Late treatment failures were treated with AL.

\section{Examinations and follow-up procedures}

Upon initial examination, a rapid screening procedure was established. Blood was generally taken by fingerprick method. Blood slides were prepared at initial presentation for all patients with fever or history of fever within $24 \mathrm{hrs}$, during routine examination at all study centres. Sampling and preparation of thin and thick smears was performed at each study centre by physicians and laboratory technicians, who had been trained and instructed by investigators at beginning of the study. Microscopic assessment of Giemsa-stained thin and thick blood smears confirmed P. falciparum mono-infection.

A general physical examination was performed on admission. After recruitment, patients were questioned via checklist about age, area of residency, underlying diseases, symptoms, with special emphasis on danger signs and symptoms for severe malaria and medications in the previous three weeks. If included, the treatment was given under observation and an additional blood sample was spotted on filter paper (Whatman 3MM) (day 0).

Patients returned for outpatient follow-up on day 2, 3, 7,28 and $42(+/-7)$ and on any day before day 42 if any symptoms recurred. Day 1 was not a regular study day although patients were instructed to return immediately if symptoms worsened. Each patient was asked on every visit about past (since last visit) and present symptoms, every adverse event was documented in specific case report forms and later transferred to the database. Severe adverse events had to be reported within 24 hours to the study PI and the ethical boards. During follow-up, thin and thick blood smears and blood-spotted filter papers were simultaneously prepared on every visit for further molecular investigations.

\section{Endpoints}

Primary and secondary endpoints were PCR-corrected and uncorrected cure rates on days 28 and 42. Further endpoints were treatment failures, completion of the follow-up period without treatment failure meaning adequate clinical and parasitological response (ACPR), loss to follow-up, voluntary and involuntary withdrawal from study and protocol violation. Treatment failures were categorized as early treatment failure (ETF), late clinical failure (LCF), and late parasitological failure (LPF) according to the definition of treatment failures for low to moderate transmission areas determined in the WHO protocol issued in 2006. Other secondary endpoints were defined as gametocytaemia at day 7 and day 28, parasitaemia at day 2 and day 3, and PCR-corrected reinfection rates at day 28 and day 42 .

\section{In vivo resistance definition and clearance time calculation}

Prolonged parasite presence (parasitaemia at day $3 \equiv \geq 72$ hours after treatment start) in $\geq 10 \%$ of cases after treatment with an ACT is defined as suspected resistance according to WHO [10], although this statement seems to need further evaluation. Artemisinin resistance seems to be highly unlikely if $\leq 3 \%$ patients show parasitaemia at day 3 , if initial parasite density was between 10,000$100,000 / \mu \mathrm{L}[29]$. 
The formula for parasite clearance was derived from the formula of half-life time: $\mathrm{e}^{-\lambda t}$.

$\lambda=\ln (0.5)$, constant of decay. $P_{2}=$ parasitaemia at day $2, \mathrm{P}_{0}=$ parasitaemia at day 0 .

$\mathrm{P}_{2}=\mathrm{P}_{0}\left(\mathrm{e}^{\left(-\ln (0.5) /-\mathrm{t}_{2}\right)(48)}\right)$ Correlating with $\mathrm{t}_{2}=(\ln (0.5) / \ln$ $\left.\left(\mathrm{P}_{0} / \mathrm{P}_{2}\right)\right)(-48 \mathrm{hrs})$, for calculation of half-life time $t_{2}$ between day 0 and day 2, meaning the time half the parasites were cleared every $t_{2}$ during $48 \mathrm{hrs}$. Analogue parasitaemia at day 3 and $t_{3}$.

\section{Laboratory examinations}

Thin and thick blood smears were prepared according to standard procedures. Slides were stained with $10 \%$ Giemsa for 20 mins and independently read by two microscopists. Parasite density was counted based on standard procedures. The number of asexual parasites was counted against 200 leukocytes assuming 8,000 leukocytes per $\mu \mathrm{L}$. Smears were determined to be negative only after examining 120 fields [100x ocular]. Parasite densities differing by more than $10 \%$ between microscopists were controlled by a third microscopist, whose result was final.

DNA from dried blood spots on Whatman filter paper (3MM) was extracted by Chelex method as described elsewhere [30]. DNA from all samples from day 0, day 42 and from all treatment failures (recurrent parasitaemia) was extracted.

Molecular genotyping was performed by polymerase chain reaction (PCR) from all samples from day 0 and all treatment failures. Therefore, sequences of parasite genes coding for the polymorphic proteins glutamaterich protein (GLURP) and merozoite surface proteins 1 and 2 (MSP-1 and MSP-2) were amplified by nested PCR assays. Plasmodium falciparum isolates were obtained at day 0 and any day of possible treatment failure up to day 42 of follow-up. PCR products of the paired samples were compared. If any pre-treatment alleles differed from post-treatment alleles, a new infection was considered; if no new allele in pre- and posttreatment samples occurred, a recrudescence was assumed [31,32].

Species amplification was performed by conventional nested PCR described by Snounou [33]. The species from all samples from day 0 , day 42 and from all treatment failures was determined.

For detection of mature gametocytes, the amplification of Pfs 25 mRNA by real-time quantitative nucleic acid sequence based amplification (QT-NASBA) was chosen as target sequence as described elsewhere [34,35]. RNA extraction was performed with using NucliSens ${ }^{\circledR}$ - easyMAG $^{\circledR}$ Lysis Buffer. Filter paper was cut out and rocked at $150 \mathrm{rpm}$ for $30 \mathrm{mins}$ at room temperature, following the original RNA extraction method described by Boom and colleagues [36]. The solution was centrifuged at
$1,500 \mathrm{~g}$ for $5 \mathrm{mins}$, the filter paper removed and $50 \mu \mathrm{l}$ of silica solution was added to the solution. The extraction was made with the NucliSens ${ }^{\circledR}$ - mimiMAG ${ }^{\text {тм }}$ unit (bioMérieux SA, Lyon, France) according to the manufacturer's manual. Amplification was performed using NucliSENS EasyQ ${ }^{\circledR}$ Basic Kit (bioMérieux Bv, RM Boxtel, The Netherlands) according to the manufacturer's manual and as described recently [35]. Microscopically gametocyte-positive and a random selection of $10 \%$ of microscopically gametocyte-negative samples were chosen.

Filter paper samples were stored at room temperature until transport to Germany (one to 12 months) and then stored at $-20^{\circ} \mathrm{C}$. DNA extraction was performed after arrival of the samples in Germany. RNA extraction for QT-NASBA was conducted about 18 months later.

\section{Data management and statistical analysis}

Data forms were checked regularly by the primary study coordinator at the site. Double data entry in Excel (Microsoft Office 2007) was independently performed in Ethiopia and Germany. All data are subject to the data protection and were processed anonymously. Data were transferred to STATA, version 11, (Statacorp, 2009) and analysis was performed. The traditional "per-protocol" and "intention-to-treat" approach were applied. Logistic regression models were used for a multivariate analysis of outcome variables (primary and secondary endpoints) and independent variables (age, gender, site, season, weight, height). Comparison between two groups was calculated by the Student's $t$-test or Wilcoxon rank sum test for continuous variables and the $\chi^{2}$ test or Fisher's exact test for categorical variables. Results were considered significant if $\mathrm{p}$-values were $<0.05$.

The anticipated population proportion of clinical and parasitological failures was estimated based upon the data of a study at Jimma Hospital in 2006 [37]. The failure rate in a 42-day course was $0 \%$; one symptomatic parasitaemia occurred on day 70 that seemed to be a recrudescence. Compared with other studies showing failure rates of about $5 \%$, a failure rate of maximum $10 \%$ was chosen [10]. Assuming follow-up losses and withdrawals during a follow-up period longer than 14 days of $20 \%$ and ACPR of $90 \%$, a sample size of $\mathrm{n}=400$ was chosen to result in a confidence interval from $86.71-93.29 \%$ on a $95 \%$ confidence level. Due to screening failures, the resulting confidence interval was $86.68 \%-93.32 \%$.

\section{Results}

\section{Baseline data}

The study was implemented in routine diagnostic of the outpatient service (Figure 1). From 20,459 patients with fever, thin and thick smears were taken for malaria diagnosis (official numbers from the health centres for the 


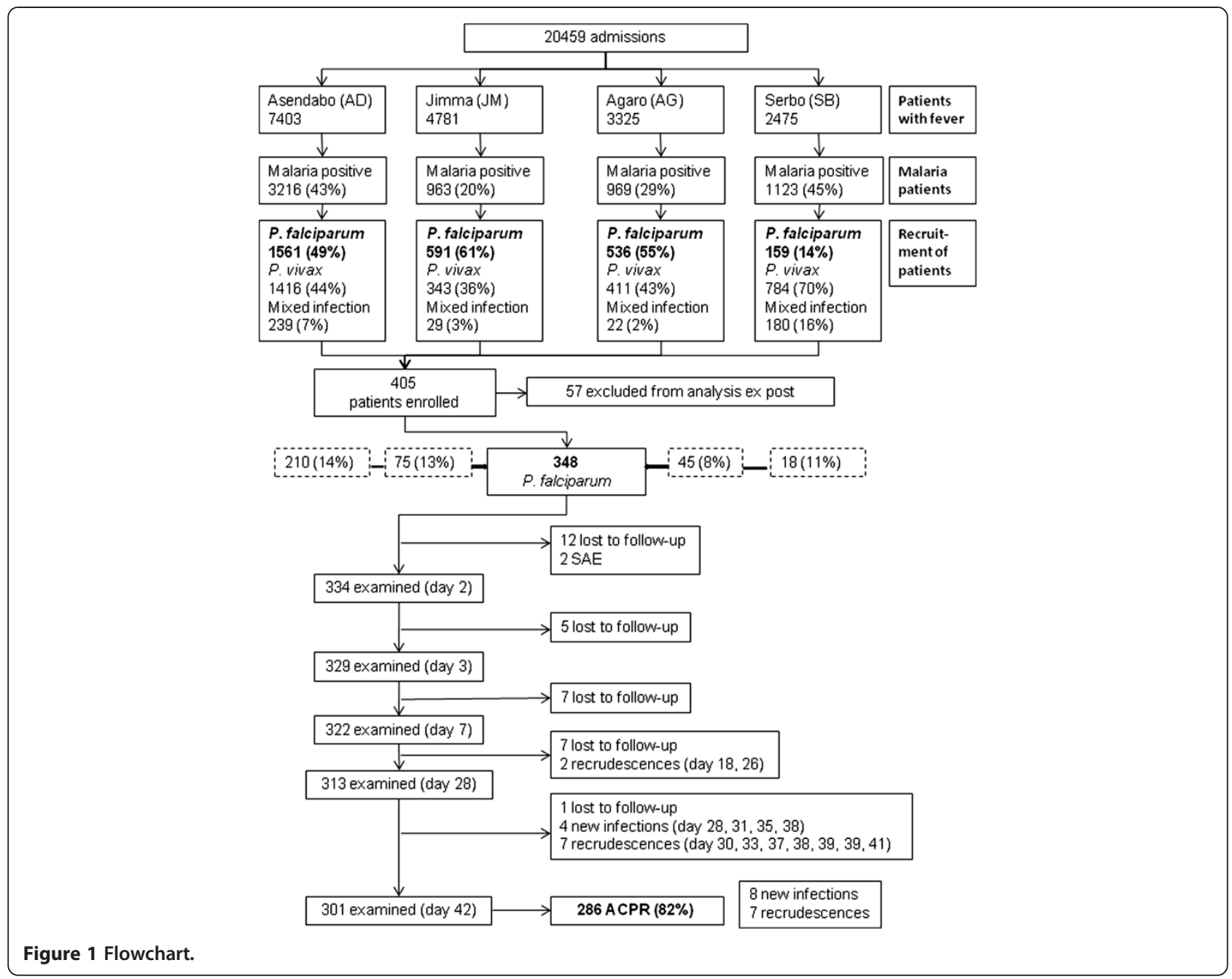

study period). Thirty-one percent (range 20-45\%) had malaria among these were 2,847 P. falciparum monoinfections. Treatment of uncomplicated malaria is on an outpatient basis. Eligibility for the study was limited to residency near to the health posts due to the follow-up schedule of the study. A total of 405 patients with uncomplicated $P$. falciparum malaria were finally enrolled from four different sites in Jimma Zone between November 2008 and January 2009 and from August to December 2009. The study was planned to be completed during the first sampling period 2008/2009 but sample collection had to be extended until end of 2009 due to weather conditions and logistics. Only $23 \%$ were recruited during the first season. Reasons for non-eligibility were age below one year, underlying chronic diseases, refusal, pregnancy, off-limits parasitaemia and in over 90\% residency too far away from the health centres.

Sixty percent of the patients were from Asendabo, 22\% from Jimma, 13\% from Agaro and 5\% from Serbo
(Table 1); 60\% were male; mean age was 17 years $(95 \%$ CI 15.7-18.5, range 1-81). Half of the patients were under 16 years old, only $13 \%$ over 50 . Children between one and five years were equally distributed between sites. Mean parasitaemia was significantly higher in infants and children under six years $(\mathrm{P}=0.004)$. Two-thirds of the patients had a parasitaemia between 5,000-40,000/ $\mu \mathrm{L}$, only $5 \%$ over $70,000 / \mu \mathrm{L}$. Gametocyte prevalence at recruitment was similar in both age groups; mean gametocytaemia was significantly lower in patients under six years $(\mathrm{P}=0.036)$.

Fifty-seven patients were excluded from the final analysis because PCR results showed mixed infection with $P$. vivax (51) or negative results (six). Re-evaluation of the slides confirmed the molecular results in some cases but sub-microscopic parasitaemia of $P$. vivax was assumed in most mixed infections. Molecularly negative samples showed parasitaemia less than $1,000 / \mu \mathrm{L}$ in microscopy. All cases with mixed infections showed adequate clinical and parasitological response at day 42 . 
Table 1 Baseline characteristics of enrolled patients, all patients (A) and stratified by age group (B, under five years and over five years)

\begin{tabular}{|c|c|c|c|c|}
\hline \multicolumn{5}{|l|}{$A$} \\
\hline Baseline characteristics & $n=348$ & $95 \% \mathrm{Cl}$ & \multicolumn{2}{|l|}{ range } \\
\hline Mean age (years) & 17.1 & $15.7-18.5$ & \multicolumn{2}{|l|}{$1-81$} \\
\hline Female (\%) & 40.2 & $35.0-45.4$ & \multicolumn{2}{|l|}{-} \\
\hline Mean temperature $\left({ }^{\circ} \mathrm{C}\right)$ & 38.1 & $38.0-38.2$ & \multicolumn{2}{|l|}{$35.6-40.6$} \\
\hline $\begin{array}{l}\text { Geometric mean parasite } \\
\text { density }\end{array}$ & 9,720 & $8,760-10,785$ & \multicolumn{2}{|c|}{$1,000-99,932$} \\
\hline Gametocytes present (\%) & 9.5 & $6.4-12.6$ & \multicolumn{2}{|l|}{-} \\
\hline GM gametocyte density & 1,305 & $899-1,894$ & \multicolumn{2}{|l|}{$40-6,798$} \\
\hline B & \multicolumn{2}{|c|}{$<\mathbf{5}$ years } & \multicolumn{2}{|c|}{$>5$ years } \\
\hline Baseline characteristics & $n=64$ & $95 \% \mathrm{Cl}$ & $\mathrm{n}=\mathbf{2 8 4}$ & $95 \% \mathrm{Cl}$ \\
\hline Mean age (years) & 3.3 & $2.9-3.6$ & 20.2 & $18.7-21.7$ \\
\hline Female (\%) & 46.9 & $34.3-59.4$ & 38.7 & $33.0-44.4$ \\
\hline Mean temperature $\left({ }^{\circ} \mathrm{C}\right)$ & 38.2 & $38.0-38.5$ & 38.0 & $37.9-38.1$ \\
\hline $\begin{array}{l}\text { Geometric mean parasite } \\
\text { density }\end{array}$ & 12,046 & $9,029-16,073^{1}$ & 9,262 & $8,299-10,336$ \\
\hline Gametocytes present (\%) & 9.4 & $2.0-16.7$ & 9.5 & $6.1-13.0$ \\
\hline GM gametocyte density & 435 & $82-2,320^{2}$ & 1,666 & $1,250-2,221$ \\
\hline
\end{tabular}

${ }^{1}$ Student's $t$-test, $\mathrm{P}=0.004$ for a difference in means between the two age groups.

${ }^{2}$ Wilcoxon rank sum test, $\mathrm{P}=0.036$ for a difference in means between the two age groups.

Of the remaining 348 patients, 32 (9.2\%) were lost to follow-up during the study (Figure 1). The age was equally distributed among those lost to follow-up and all other study participants. The relation between children $\leq 5$ years and elder patients was similar in both groups but relatively more boys $\leq 5$ years $(73.5 \%)$ were lost. There was no evidence for a real difference in parasitaemia between those lost to follow-up and the other participants $(\mathrm{p}>0.05)$. Those lost to follow-up after day 2 were already negative in blood slides at day 2 .

\section{Study outcome}

Until day 42, 286 patients showed an adequate clinical and parasitological response (ACPR). Twenty-eight LTF were observed during follow-up, 16 (4.6\%) were LCF and $12(3.4 \%)$ were LPF. PCR-correction showed 16 recrudescences, of which $62.5 \%$ were symptomatic, and incidence of 12 new infections. Recrudescences started on day 18 until 42 (mean: 37 days); the first new infection occurred at day 28 (mean: 39 days).

The LTF rate was barely influenced by drop outs as only one was lost to follow-up between day 28 and 42 .

For per protocol (PP) analysis, PCR-corrected cure rates at day 28 and 42 were 99.4 (95\% CI 98.5-100.0) and 94.7 (95\% CI 92.2-97.2), respectively. Uncorrected cure rates at day 28 and 42 were 99.1 (95\% CI 98.0100.0 ) and 91.1 (95\% CI 87.9-94.3), respectively (Table 2). At day 42, PCR-corrected cure rate for children $\leq 5$ years was 90.6 (95\% CI 82.4-98.7) compared to 95.6 (95\% CI 93.0-98.2) in older patients, but the difference lacked significance $(\mathrm{P}=0.17)$ (Additional file 1$)$.

For the intention to treat analysis (ITT), uncorrected cure rates at day 28 and 42 were 89.7 (95\% CI 86.4-92.9) and 82.2 (95\% CI 78.1-86.2), respectively; PCR-corrected cure rates at day 28 and 42 were 89.9 (95\% CI 86.7-93.1) and 85.1 (95\% CI 81.3-88.9), respectively (Table 2).

Table 2 Outcome at day $\mathbf{2 8}$ and $\mathbf{4 2}$ for all patients

\begin{tabular}{|c|c|c|}
\hline Treatment outcome & Day 28 & Day 42 \\
\hline Lost to follow-up (\%) & $31 / 348(8.9)$ & $32 / 348(9.2)$ \\
\hline Inability to tolerate oral treatment ${ }^{1}(\%)$ & 2/348 (0.6) & $2 / 348(0.6)$ \\
\hline Early treatment failure (\%) & 0 & 0 \\
\hline Late treatment failure (\%) & $3 / 348(0.9)$ & 28/348 (8.0) \\
\hline Late clinical failure (\%) & $3 / 348(0.9)$ & $16 / 348(4.6)$ \\
\hline Late parasitological failure & 0 & $12 / 348(3.4)$ \\
\hline ACPR & $312 / 348(89.7)$ & $286 / 348(82.2)$ \\
\hline Infection with different species (\%) & 0 & 0 \\
\hline New infection P. falciparum (\%) & $1 / 348(0.3)$ & $12 / 348(3.4)$ \\
\hline Recrudescences (\%) & 2/348 (0.6) & $16 / 348(4.6)$ \\
\hline Cure rate per protocol, PCR-uncorrected (\%, 95\% Cl) & $312 / 315(99.1,98.0-100.0)$ & 286/314 (91.1, 87.9-94.3) \\
\hline Cure rate per protocol, PCR-corrected (\%, 95\% Cl) & $312 / 314(99.4,98.5-100.0)$ & 286/302 (94.7, 92.2-97.2) \\
\hline Cure rate intention-to-treat, PCR-uncorrected (\%, 95\% Cl) & $312 / 348(89.7,86.4-92.9)$ & $286 / 348(82.2,78.1-86.2)$ \\
\hline Cure rate intention-to-treat, PCR-corrected $(\%, 95 \% \mathrm{Cl})$ & $312 / 347(89.9,86.7-93.1)$ & 286/336 (85.1, 81.3-88.9) \\
\hline
\end{tabular}

${ }^{1}$ Those patients defined as "adverse event requiring change in anti-malarial therapy prior to completion of full dose of study drug". They were included in the intention-to-treat analysis but excluded from the per-protocol analysis. 


\section{Fever clearance}

Fever was more rapidly cleared in patients $>5$ years (Table 3).

\section{Parasite clearance and associated variables}

Six patients (1.9\%) showed a prolonged duration till clearance with parasites still present at day 2 (four patients) - mean half-life $t_{2}$ of 9.9 hours [range 7.3-13.9] - and at day 3 (two patients) - mean half-life $t_{3}$ of 21.4 hours [range 11.9-30.9]. All parasites were cleared at day 7. For those without parasitaemia at day 2 or later, mean half-life of $<2.9-4.8$ hours can be assumed, considering 1,000-100,000 parasite/ $\mu \mathrm{L}$ at admission. Values are only an approximation ( \pm 8 hours) as it was not possible to take the blood exactly 48 or 72 hours later (Table 3).

Mean age of late responders ( $>48 \mathrm{hrs}$ ) was 11 years [range 1.3-22.0]. They were from all clusters; two were $\leq 5$ years, underweight and stunted. Initial parasitaemia at day 0 was below $10,000 / \mu \mathrm{L}$ (mean parasitaemia 7,058 / $\mu \mathrm{L}$, range $3,800-8,800 / \mu \mathrm{L}$ ) in all six patients. Criteria for an early treatment failure, defined by WHO [28], were not fulfilled. Parasitaemia at day 3 was $20 \%$ and $2 \%$ of day 0 , respectively. Both patients with parasites at day 3 showed ACPR at day 42.

However, there was evidence for a strong association of prolonged parasite presence $(>48 \mathrm{hrs})$ with recrudescence $(\mathrm{OR} 9.5,95 \%$ CI 1.6-57.5, $\mathrm{P}=0.01)$, meaning a recrudescence was 9.5 times more likely when parasites were cleared after day 2 (Additional file 2).

Parasite density, on the other hand, seemed to have no influence on recrudescent infections. Recrudescences presented with a geometric mean parasitaemia of 8,665 / $\mu \mathrm{L}(95 \%$ CI 6,619-11,344/ $\mu \mathrm{L})$ at day 0 . Mean geometric

Table 3 Fever and parasitaemia clearance

\begin{tabular}{llll}
\hline Outcome & $\begin{array}{c}<=5 \text { years } \\
\text { of age }\end{array}$ & $\begin{array}{l}>\mathbf{5} \text { years } \\
\text { of ag }\end{array}$ & Total \\
\hline Fever, n/N (\%) & $1 / 60(1.7)$ & $10 / 274(3.7)$ & $11 / 334(3.3)$ \\
Day 2 & 0 & $3 / 269(1.1)$ & $3 / 329(0.9)$ \\
Day 3 & $2 / 60(3.3)$ & $4 / 274(1.4)$ & $6 / 334(1.8)$ \\
Microscopically determined parasitaemia, $\mathrm{n} / \mathrm{N}(\%)$ & $2 / 329(0.6)$ \\
Day 2 & 0 & $2 / 270(0.7)$ & \\
Day3 & $6 / 64(9.4)$ & $26 / 284(9.5)$ & $33 / 348(9.5)$ \\
Microscopically determined gametocytaemia, $\mathrm{n} / \mathrm{N}(\%)$ & $17 / 334(5.1)$ \\
Day 0 & $5 / 60(8.3)$ & $12 / 274(4.4)$ & $10 / 329(3.0)$ \\
Day 2 & $4 / 60(6.7)$ & $6 / 269(1.9)$ & $7 / 322(2.2)$ \\
Day 3 & $1 / 57(1.8)$ & $6 / 265(2.3)$ & $3 / 313(1.0)$ \\
Day 7 & $1 / 54(1.9)$ & $2 / 259(0.8)$ & $2 / 301(0.7)$ \\
Day 28 & $1 / 53(1.9)$ & $1 / 258(0.4)$ & \\
Day 42 (37) & & & \\
\hline
\end{tabular}

parasitaemia of 286 patients with ACPR was $9,431 / \mu \mathrm{L}$ (95\% CI 8,402-10,586/ $\mu \mathrm{L})$. There was no evidence of a real difference between these groups $(\mathrm{P}=0.80)$.

\section{Microscopic gametocyte clearance and associated variables}

Gametocyte prevalence was $9.5 \%$ by microscopy. Eighty percent reduction could be observed between day 0 and day 7 (Additional file 3). Only two patients developed microscopic gametocytaemia after treatment start (Table 3). Three patients had gametocytes on day 28. They were already positive on day 0 and below 13 years (median 7 years).

The association of recrudescences (after day 28) with gametocytaemia at day 28 was significant (OR 38.5, 95\% CI 3.1-485.4, $\mathrm{P}<0.01$ ) (Additional file 2). There was also evidence for a strong association of prolonged parasite presence with gametocytaemia on day 7 and day 28 (OR 10.1, 95\% CI 1.0-99.8; P $=0.05$ and OR 20.4, 95\% CI 1.4309.8; $\mathrm{P}<0.03$, respectively). Controlled for site, gender, age and parasite density, the prevalence of gametocytes at any point in time between day 0 to day 42 was more likely in season 1 than in season 2 (OR 0.47, 95\% CI $0.22-1.01, \mathrm{P}=0.05)$.

\section{Molecular and submicroscopic investigations}

Species PCR was done for all samples on day 42 with 12 (4.2\%) of the 286 patients with ACPR being positive for $P$. falciparum, no parasites were microscopically found. Therefore, sub-microscopic levels of either asexual or sexual parasitaemia can be assumed.

In 32 randomly chosen microscopically negative samples from day 0 , the gametocyte-specific $P f s 25$-mRNA could still be detected in $22(70 \%)$ of the samples by QT-NASBA. That means that microscopy revealed less than one of seven of the prevalence of gametocytes in these 32 patients. As storage duration and conditions of filter-paper samples as well as blood spot sizes were not standardized, molecular quantification of gametocytes was not possible.

\section{Symptoms, adverse events and pregnancy}

Most patients had fever, headache, shivering and nausea at admission. Abdominal pain and diarrhoea were less frequent (Additional file 4): $2.9 \%$ of the patients had adverse events (AE) on day 2 and $3.3 \%$ on day 3 with abdominal pain and diarrhoea being the most prevalent (Additional file 5). Two infants with high parasitaemia $(>95,000 / \mu \mathrm{L})$ were recruited but did not tolerate oral treatment. After re-dosing and repeated vomiting, the infants were referred to the ward for intravenous treatment; one died the same day. Both patients were reported as serious adverse events (SAE) but classified as "adverse event requiring change in anti-malarial 
therapy prior to completion of full dose of study drug". A rapid aggravation of symptoms during the enrolment procedure was suspected, no signs of severe malaria were noticed at admission. Both children received IV quinine, as oral therapy was not tolerated.

No further SAE occurred and no pregnancy (exclusion criteria) was reported.

\section{Discussion}

Of all 348 patients, $91.3 \%$ responded clinically and parasitically adequately to the treatment until day 42 . Adverse events were expectedly mild to moderate with low prevalence. Abdominal pain and diarrhoea were the most frequent symptoms.

The PCR-corrected failure rate showed incidence rates of $3.8 \%$ for new infections and $5.1 \%$ for recrudescences at day 42. No early treatment failure occurred. Compared to a trial in 2006, these data show a slight increase in recurrent parasitaemia as none had been detected until day 42 [37].

Other Ethiopian studies detected also high cure rates but most had a follow-up period of 28 days only [1923]. In this study, the PCR-corrected cure rate per protocol at day 28 was $99.4 \%$ (95\% CI 98.5-100.0). This result emphasizes the need for follow-up periods of at least 42 days. Most recrudescences occurred after day 28. The recrudescence rate in children $\leq 5$ years was surprisingly high with $9.4 \%$. None of the children with recrudescences had diarrhoea or vomiting at admission or during therapy. Apart from limitations discussed in the next paragraph, the weight-adapted drug dose is non-linear but stepwise. Two of the five children with LTF had calculated drug doses of less than $2 \mathrm{mg} / \mathrm{kg}$ and $12 \mathrm{mg} / \mathrm{kg}$ body weight, respectively. Furthermore, weight-adapted linear or non-linear doses might not correlate with plasma drug levels in children in general.

Unfortunately, there are several limitations concerning this study. Drug levels were not tested and only the first drug intake was observed. As absorption is fatdependent, fatty food was provided and patients were precisely instructed how and when to take the rest of the medication. However, intake was not directly controlled and absorption individually varies, also impaired by symptoms like diarrhoea [38]. Therefore, recrudescences and also the prolonged parasite presence could be due to insufficient drug levels. The advantage of this study is the closer approach to a real life situation.

Another limitation is the method of genotyping. Differentiation of recrudescences and re-infections by PCR is unprecise, especially in low to moderate transmission areas with a limited diversity of strains [32]. Similar strains, defined as recrusdescence, could be new infections instead. The recrudescence rate given might be lower in reality.

Artemether has a very short half-life of about one hour. Lumefantrine has a half-life of three to six days and is able to clear the remaining parasites and to prevent recurrent parasitaemia [39,40]. The first recrudescence was observed early at day 18 . However, due to limitations mentioned above, other reasons than strain resistance might have caused recrudescences. New infections seemed to be efficiently prevented in the first four weeks.

Six patients showed prolonged presence of parasites detectable still at day 2 and day 3, the calculated half-life time was prolonged. As stated above, this could have been caused by low drug levels. Another theory is the hypothesis of a dormant non-responding parasite stage causing delayed susceptibility to drugs [41,42]. This might be responsible for late clearance rates, though genetic analysis revealed a heritable trait at the Cambodia-Thailand border indicating changes in parasite genetics and a potential for spread [15]. Controlled ACT studies with measurement of drug levels and observed drug intake should be conducted in Africa to examine drug efficacy and clearance rates.

The low number of recrudescences and prolonged parasite presence $(>48 \mathrm{hrs}$ ) impaired multivariate analysis. Risk factors for these outcomes could not be determined. There was strong evidence for an association of both outcomes but the result has to be considered with caution as the sample size is low. However, an association of recrudescence and prolonged parasite presence (72 hrs) was described recently [29]. Parasite clearance data from 18,699 patients with uncomplicated falciparum malaria were analysed. Blood smear result on day 3 was reported as a good predictor of subsequent treatment failure in low- and moderatetransmission areas. The risk of recrudescence was $<5 \%$ if parasitaemia was cleared before day 3, patients with parasitaemia on day 3 on the other hand had a significantly higher risk in low-transmission areas (12\%) and in moderate-transmission areas (34\%) to develop recrudescences. Less pronounced but still significant was the association of parasitaemia at day 2 and recrudescences in moderate transmission areas.

This association was observed in the underlying study. Recurrent parasitaemia occurred in two patients with parasites at day $2(\mathrm{P}=0.01)$. Prevalence of patients with parasites at day 3 was $0.6 \%(2 / 348)$ only. Even if adequate drug levels could be assumed, tolerance to artemether would not be suspected. Artemisinin resistance seems highly unlikely if prevalence of patients with parasites at day 3 is $<3 \%$ [29].

Further analysis indicated a correlation of (prolonged) presence of gametocytes with prolonged parasitaemia 
and recrudescences. Sample size was low as mentioned but associations seemed plausible. Gametocytes have more opportunities to develop if parasite presence is prolonged and sub-microscopic level as with recrudescences seem to suffice for gametocyte production.

Interestingly, gametocytaemia was more frequent in season 2008 than 2009. The broad introduction of $\mathrm{ACT}$ in this region might have influenced the gametocyte carriage in the population. ACT clears gametocytes better than S/P, the previous first-line drug. ACT is supposed to destroy the early stages of gametocytes $[16,43]$. The carrier reservoir might be reduced since introduction of ACT.

A random selection of samples showed very high levels of gametocytaemia with QT-NASBA technique compared to microscopy. Therefore, higher submicroscopic levels have to be assumed on recruitment as well as after treatment. Recent studies showed a difference of factor 3-6 between microscopic and submicroscopic gametocyte prevalence $[44,45]$. Less than one gametocyte per $\mu \mathrm{L}$ is sufficient to infect a mosquito [43]. Gametocytes detected by microscopy were rapidly reduced under AL in this study but $2.2 \%$ of carriers remained positive until day 7. A much higher proportion of submicroscopic gametocytaemia can be assumed. ACT seems to have less or no effect on mature gametocytes $[16,43]$. Therefore, stage V gametocytes, if already developed at start of therapy, might circulate for two to three weeks. The reservoir is a permanent risk for further transmission and should be measured in clinical trials as well as in population-based studies with recently published methods [35].

Further, an additional drug for gametocyte clearance should be considered in the area. A single dose of primaquine, best given at day 8 , might be sufficient for gametocyte clearance [46]. Unfortunately, prevalence of G6PD enzyme deficiency is unknown. As tests are not yet available in this area, the application of primaquine remains ethically problematic, although single-dose treatment seems acceptable [43].

Interestingly, P. falciparum mono-infection occurred in only half of the malaria cases in the study area. Prevalence of mixed infections with $P$. vivax and infections with $P$. vivax alone was high and has increased over the last years [[9], pers comm with health centres in Jimma and surrounding]. In Serbo, $70 \%$ of the malaria patients were infected with $P$. vivax only. However, there was surprisingly no re-infection with $P$. vivax observed. One explanation for this and the very few re-infections with $P$. falciparum might be that new bed nets were given free of charge by the government to every patient visiting the health centres.

Plasmodium vivax infections diagnosed by PCR and afterwards excluded from analysis showed ACPR in all cases, no relapses occurred. Data on P. vivax and AL are unavailable in this area. Chloroquine is still first-line treatment of $P$. vivax. A recent study from another area from Ethiopia observed treatment with $\mathrm{AL}$ and $\mathrm{CQ}$ in vivax malaria. Recurrent parasitaemia rates were 19\% and $8 \%$, respectively, although, the evening dose of $\mathrm{AL}$ was not observed [47]. Studies from Asia reported higher recurrence rates with $\mathrm{AL}$ compared to $\mathrm{CQ}$ or dihydro-artemisinin/piperaquine as well $[48,49]$. However, relapses seem to occur in Ethiopia later than in Asia [43] and might be expected after day 42.

\section{Conclusion}

AL showed good effectiveness in Jimma. The overall PCR-corrected cure rate per protocol was $94.7 \%$, resulting in a recrudescence rate of below $10 \%$. The PCRcorrected per-protocol failure rate for children $\leq 5$ years was $9.4 \%$. Low drug levels due to malabsorption and dosing problems in children could be responsible. To monitor closely the further development of drug susceptibility in this area, a continuous surveillance should be established and controlled ACT studies with measurement of drug levels and observed drug intake should be conducted.

\section{Additional files}

\begin{abstract}
Additional file 1: Outcome at day $\mathbf{4 2}$ stratified in age groups. Description: The table shows the outcome at day 42 stratified in the age groups $>5$ years and $\leq 5$ years. The cure rates are in general lower for the under five years old children but the difference is not significant.
\end{abstract}

Additional file 2: Association with outcome recrudescence (ACPR \& Recrudescences, $\mathbf{n}=\mathbf{3 0 2}$ ). Description: The data show a multi-variate analysis with the outcome recrudescences as dependent variable and independent variables like parasitaemia at day 0 , gametocytaemia over time, and parasite clearance, controlled for age and gender. Delayed clearance and gametocytaemie at day 28 were associated with recrudescences. There was evidence for a strong association $(P \leq 0.01)$ but confidence intervals were wide.

Additional file 3: Clearance of microscopically detected gametocytes over time. Description: Clearance of microscopically detected gametocytes during follow-up in both age groups and overall. Clearance in older patients seemed to be faster than in children below 6 years of age.

Additional file 4: Symptoms at recruitment $(n=348)$. Description: The data show all symptoms presented at admission with absolute and relative frequencies and duration. Fever, headache and shivering were the leading symptoms.

Additional file 5: Adverse events (AE) during follow-up. Description: The table presents all adverse events reported until day 7. Abdominal pain was the most reported AE. Prevalence was overall low.

\section{Competing interests}

The authors declare that they have no competing interests.

\section{Authors' contributions}

TE coordinated the clinical study in Ethiopia, participated in the molecular genetic studies and helped draft the manuscript. NA, KB and SF participated in and carried out the clinical studies. AW and MP established the QT-NASBA 
technology, carried out the gametocyte analysis and revised the manuscript. TL participated in study design and coordination and drafted the manuscript. NB-R designed the study protocol, was PI of the study, carried out the molecular genetic studies as well as the sequence alignments, conducted the statistical analysis and drafted the manuscript. All authors read and approved the final manuscript.

\section{Acknowledgements}

We thank all study participants, clinicians and laboratory workers at the study sites in Ethiopia as well as the laboratory personnel in Munich for technical assistance. Our special thanks go to the Friedrich Baur Foundation for funding and to the organisation "Menschen für Menschen" for logistic support. TE was supported by a research grant from the German Academic Exchange Service (DAAD).

\section{Author details}

'Division of Infectious Diseases and Tropical Medicine, Medical Center of the University of Munich (LMU), Leopoldstrasse 5, 80802 Munich, Germany. ${ }^{2}$ Department of Medical Laboratory Sciences and Pathology, Jimma University (JU), Jimma, Ethiopia. ${ }^{3}$ Jimma Zone Health Bureau, Jimma, Ethiopia. ${ }^{4}$ Department of Internal Medicine, Jimma University (JU), Jimma, Ethiopia. ${ }^{5}$ Max von Pettenkofer-Institute of Hygiene and Medical Microbiology, Munich, Germany. ${ }^{6}$ German Centre for Infection Research (DZIF) at LMU, Munich, Germany.

Received: 31 May 2012 Accepted: 23 July 2012

Published: 23 July 2012

\section{References}

1. Federal Democratic Republic of Ethiopia, Ministry of Health: Malaria diagnosis and treatment guidelines for health workers in Ethiopia. 2nd edition. Addis Ababa: Ministry of Health; 2004.

2. World Health Organization: WHO Guidelines for the treatment of malaria. Geneva: WHO; 2006

3. van Vugt M, Looareesuwan S, Wilairatana P, McGready R, Villegas L, Gathmann I, Mull R, Brockman A, White NJ, Nosten F: Artemetherlumefantrine for the treatment of multidrug-resistant falciparum malaria. Trans R Soc Trop Med Hyg 2000, 94:545-548.

4. White NJ: Qinghaosu (artemisinin): the price of success. Science 2008 320:330-334.

5. Omari AA, Gamble C, Garner P: Artemether-lumefantrine (six-dose regimen) for treating uncomplicated falciparum malaria. Cochrane Database Syst Rev 2005, 4:CD005564.

6. Krishna S, Uhlemann AC, Haynes RK: Artemisinins: mechanisms of action and potential for resistance. Drug Resist Updat 2004, 7:233-244.

7. White NJ: Qinghaosu in combinations. Med Trop (Mars) 1998 58(Suppl 3):85-88

8. Abeku TA, van Oortmarssen GJ, Borsboom G, de Vlas SJ, Habbema JD: Spatial and temporal variations of malaria epidemic risk in Ethiopia: factors involved and implications. Acta Trop 2003, 87:331-340.

9. Alemu A, Tsegaye W, Golassa L, Abebe G: Urban malaria and associated risk factors in Jimma town, south-west Ethiopia. Malar J 2011, 10:173.

10. Ringwald P, Barrette A, Fairhurst R, Dondorp A, Kachur P, MacArthur J, Slutsker L, Plowe C, Dye C, Mendis K, Newman R, Olumese P, Sillah J, Warsame M: Global report on antimalarial drug efficacy and drug resistance: 2000-2010. Geneva: World Health Organization; 2011.

11. Noedl H, Se Y, Schaecher K, Smith BL, Socheat D, Fukuda MM, Artemisinin Resistance in Cambodia 1 (ARC1) Study Consortium: Evidence of artemisinin-resistant malaria in western Cambodia. N Engl J Med 2008 359:2619-2620.

12. Dondorp AM, Nosten F, Yi P, Das D, Phyo AP, Tarning J, Lwin KM, Ariey F, Hanpithakpong W, Lee SJ, Ringwald P, Silamut K, Imwong M, Chotivanich K, Lim P, Herdman T, An SS, Yeung S, Singhasivanon P, Day NP, Lindegardh N, Socheat $D$, White $\mathrm{NJ}$ : Artemisinin resistance in Plasmodium falciparum malaria. N Engl J Med 2009, 361:455-467.

13. Noedl $H$, Socheat $D$, Satimai W: Artemisinin-resistant malaria in Asia. $N$ Engl J Med 2009, 361:540-541.

14. Dondorp AM, Fairhurst RM, Slutsker L, Macarthur JR, Breman JG, Guerin PJ, Wellems TE, Ringwald P, Newman RD, Plowe CV: The threat of artemisininresistant malaria. N Engl J Med 2011, 365:1073-1075.
15. Anderson TJ, Nair S, Nkhoma S, Williams JT, Imwong M, Yi P, Socheat D, Das D, Chotivanich K, Day NP, White NJ, Dondorp AM: High heritability of malaria parasite clearance rate indicates a genetic basis for artemisinin resistance in western Cambodia. J Infect Dis 2010, 201:1326-1330.

16. Makanga M, Krudsood S: The clinical efficacy of artemether/lumefantrine (Coartem). Malar J 2009, 8(Suppl 1):5.

17. Barnes Kl, Chanda P, Ab Barnabas G: Impact of the large-scale deployment of artemether/lumefantrine on the malaria disease burden in Africa: case studies of South Africa, Zambia and Ethiopia. Malar J 2009, 8(Suppl 1):8.

18. World Health Organization: Global plan for artemisinin resistance containment (GPARC). Global Partnership to Roll Back Malaria \& Geneva: World Health Organization; 2011.

19. Seboxa T, Mao P, Pinchouk N, Anbessie J, Alemu H, Diro E: ArtemetherLumefantrin (Coartem) and artesunate with sulfadoxine-pyrimethamine therapeutic efficacy in the treatment of uncomplicated malaria at Gilgel Gibe II (GGII) South-Western Ethiopia. Ethiop Med J 2010, 48:285-291.

20. Kefyalew T, Animut A, Tamene T, Jima D, Hailemariam A, Legesse M: Efficacy of six-dose regimen of artemether-lumefantrine for the treatment of uncomplicated falciparum malaria, three years after its introduction into Ethiopia. Parasite 2009, 16:129-134.

21. Kinfu G, Gebre-Selassie S, Fikrie N: Therapeutic efficacy of artemetherlumefantrine for the treatment of uncomplicated Plasmodium falciparum Malaria in Northern Ethiopia. Malar Res Treat 2012, 2012:548710.

22. Assefa A, Kassa M, Tadese G, Mohamed H, Animut A, Mengesha T: Therapeutic efficacy of artemether/lumefantrine (Coartem(R)) against Plasmodium falciparum in Kersa, South West Ethiopia. Parasit Vectors 2010, 3:1.

23. Hwang J, Alemayehu BH, Hoos D, Melaku Z, Tekleyohannes SG, Teshi T, Birhanu SG, Demeke L, Gobena K, Kassa M, Jima D, Reithinger R, Nettey H, Green M, Malone JL, Kachur SP, Filler S: In vivo efficacy of artemetherlumefantrine against uncomplicated Plasmodium falciparum malaria in Central Ethiopia. Malar J 2011, 10:209

24. Bassat Q, González R, Machevo S, Nahum A, Lyimo J, Maiga H, Mårtensson A, Bashraheil M, Ouma P, Ubben D, Walter V, Nwaiwu O, Kipkeu C, Lefèvre G, Ogutu B, Menéndez C: Similar efficacy and safety of artemetherlumefantrine (Coartem ${ }^{\circledR}$ ) in African infants and children with uncomplicated falciparum malaria across different body weight ranges. Malar J 2011, 10:369.

25. Kamugisha E, Jing S, Minde M, Kataraihya J, Kongola G, Kironde F, Swedberg G: Efficacy of artemether-lumefantrine in treatment of malaria among under-fives and prevalence of drug resistance markers in lgombeMwanza, north-western Tanzania. Malar J 2012, 11:58.

26. Four Artemisinin-Based Combinations (4ABC) Study Group: A head-to-head comparison of four artemisinin-based combinations for treating uncomplicated malaria in African children: a randomized trial. PLOS Med 2011, 8:e1001119.

27. Borrmann S, Sasi P, Mwai L, Bashraheil M, Abdallah A, Muriithi S, Frühauf $H$ Schaub B, Pfeil J, Peshu J, Hanpithakpong W, Rippert A, Juma E, Tsofa B, Mosobo M, Lowe B, Osier F, Fegan G, Lindegårdh N, Nzila A, Peshu N, Mackinnon M, Marsh K: Declining responsiveness of Plasmodium falciparum infections to artemisinin-based combination treatments on the Kenyan coast. PLoS One 2011, 6:e26005.

28. World Health Organization: Assessment and monitoring of antimalarial drug efficacy for the treatment of uncomplicated falciaprum malaria. Geneva: WHO/HTM/RBM; 2003.

29. Stepniewska K, Ashley E, Lee SJ, Anstey N, Barnes Kl, Binh TQ, D'Alessandro U, Day NP, de Vries PJ, Dorsey G, Guthmann JP, Mayxay M, Newton PN, Olliaro P, Osorio L, Price RN, Rowland M, Smithuis F, Taylor WR, Nosten F, White NJ: In vivo parasitological measures of artemisinin susceptibility. $J$ Infect Dis 2010, 201:570-579.

30. Kain KC, Lanar DE: Determination of genetic variation within Plasmodium falciparum by using enzymatically amplified DNA from filter paper disks impregnated with whole blood. J Clin Microbiol 1991, 29:1171-1174

31. Snounou G, Beck HP: The use of PCR genotyping in the assessment of recrudescence or reinfection after antimalarial drug treatment. Parasitol Today 1998, 14:462-467.

32. Mugittu K, Adjuik M, Snounou G, Ntoumi F, Taylor W, Mshinda H, Olliaro P, Beck HP: Molecular genotyping to distinguish between recrudescents and new infections in treatment trials of Plasmodium falciparum malaria 
conducted in Sub-Saharan Africa: adjustment of parasitological outcomes and assessment of genotyping effectiveness. Trop Med Int Health 2006, 11:1350-1359.

33. Snounou G, Viriyakosol S, Zhu XP, Jarra W, Pinheiro L, do Rosario VE, Thaithong S, Brown KN: High sensitivity of detection of human malaria parasites by the use of nested polymerase chain reaction. Mol Biochem Parasitol 1993, 61:315-320.

34. Schneider P, Schoone G, Schallig H, Verhage D, Telgt D, Eling W, Sauerwein R: Quantification of Plasmodium falciparum gametocytes in differential stages of development by quantitative nucleic acid sequence-based amplification. Mol Biochem Parasitol 2004, 137:35-41.

35. Pritsch M, Wieser A, Soederstroem V, Poluda D, Eshetu T, Hoelscher M, Schubert S, Shock J, Loescher T, Berens-Riha N: Stability of gametocytespecific Pfs25-mRNA in dried blood spots on filter paper subjected to different storage conditions. Mal J. 2012, 11:138.

36. Boom R, Sol CJ, Salimans MM, Jansen CL, Wertheim-van Dillen PM, van der Noordaa J: Rapid and simple method for purification of nucleic acids. J Clin Microbiol 1990, 28:495-503.

37. Gürkov R, Eshetu T, Miranda IB, Berens-Riha N, Mamo Y, Girma T, Krause E, Schmidt M, Hempel JM, Löscher T: Ototoxicity of artemether/lumefantrine in the treatment of falciparum malaria: a randomized trial. Malar $J$ 2008, 7:179.

38. White NJ, van Vugt M, Ezzet F: Clinical pharmacokinetics and pharmacodynamics and pharmacodynamics of artemether-lumefantrine. Clin Pharmacokinet 1999, 37:105-125.

39. Kokwaro G, Mwai L, Nzila A: Artemether/lumefantrine in the treatment of uncomplicated falciparum malaria. Expert Opin Pharmacother 2007, 8:75-94.

40. Travassos MA, Laufer MK: Resistance to antimalarial drugs: molecular, pharmacologic, and clinical considerations. Pediatr Res 2009, 65:64R-70R.

41. Hoshen MB, Na-Bangchang K, Stein WD, Ginsburg H: Mathematical modelling of the chemotherapy of Plasmodium falciparum malaria with artesunate: postulation of 'dormancy', a partial cytostatic effect of the drug, and its implication for treatment regimens. Parasitology 2000, 121:237-246.

42. Witkowski B, Lelièvre J, Barragán MJ, Laurent V, Su XZ, Berry A, Benoit-Vical F: Increased tolerance to artemisinin in Plasmodium falciparum is mediated by a quiescence mechanism. Antimicrob Agents Chemother 2010, 54:1872-1877.

43. Bousema T, Drakeley C: Epidemiology and infectivity of Plasmodium falciparum and Plasmodium vivax gametocytes in relation to malaria control and elimination. Clin Microbiol Rev 2011, 24:377-410.

44. Shekalaghe SA, Bousema JT, Kunei KK, Lushino P, Masokoto A, Wolters LR, Mwakalinga S, Mosha FW, Sauerwein RW, Drakeley CJ: Submicroscopic Plasmodium falciparum gametocyte carriage is common in an area of low and seasonal transmission in Tanzania. Trop Med Int Health 2007, 12:547-553.

45. Bousema JT, Schneider P, Gouagna LC, Drakeley CJ, Tostmann A, Houben R, Githure JI, Ord R, Sutherland CJ, Omar SA, Sauerwein RW: Moderate effect of artemisinin-based combination therapy on transmission of Plasmodium falciparum. J Infect Dis 2006, 193:1151-1159.

46. Lawpoolsri S, Klein E, Singhasivanon P, Yimsamran S, Thanyavanich N, Maneeboonyang W, Hungerford L, Maguire J, Smith D: Optimally timing primaquine treatment to reduce Plasmodium falciparum transmission in low endemicity Thai-Myanmar border populations. Malar J 2009, 8:159.

47. Yohannes AM, Teklehaimanot A, Bergqvist Y, Ringwald P: Confirmed vivax resistance to chloroquine and effectiveness of artemether-lumefantrine for the treatment of vivax malaria in Ethiopia. Am Jrop Med Hyg 2011, 84:137-140.

48. Krudsood S, Tangpukdee N, Muangnoicharoen S, Thanachartwet V, Luplertlop N, Srivilairit S, Wilairatana P, Kano S, Ringwald P, Looareesuwan S: Clinical efficacy of chloroquine versus artemether-lumefantrine for Plasmodium vivax treatment in Thailand. Korean J Parasitol 2007, 45:111-114.

49. Ratcliff A, Siswantoro H, Kenangalem E, Maristela R, Wuwung RM, Laihad F, Ebsworth EP, Anstey NM, Tjitra E, Price RN: Two fixed-dose artemisinin combinations for drug-resistant falciparum and vivax malaria in Papua, Indonesia: an open-label randomised comparison. Lancet 2007, 369:757-765.

\section{doi:10.1186/1475-2875-11-240}

Cite this article as: Eshetu et al:: Open-label trial with artemetherlumefantrine against uncomplicated Plasmodium falciparum malaria three years after its broad introduction in Jimma Zone, Ethiopia. Malaria Journal 2012 11:240.

\section{Submit your next manuscript to BioMed Central and take full advantage of:}

- Convenient online submission

- Thorough peer review

- No space constraints or color figure charges

- Immediate publication on acceptance

- Inclusion in PubMed, CAS, Scopus and Google Scholar

- Research which is freely available for redistribution

Submit your manuscript at www.biomedcentral.com/submit
C Biomed Central 